\title{
Aparelhos intraorais e sua eficácia no tratamento de pacientes com ronco primário e com síndrome da apneia e hipopneia obstrutiva do sono (SAOS): uma revisão de literatura
}

\section{Intraoral appliances and their effectiveness in treating patients with primary snoring and obstructive sleep apnea-hypopnea syndrome (SAOS): a literature review}

\author{
Poliana Lima Bastos* \\ Millena Teles Portela de Oliveira* \\ Guilherme Salles Ottoboni*** \\ Morgana Rocha Pontes ${ }^{* * * *}$ \\ Jorge Machado Caram ${ }^{* * * * * *}$
}

\section{Resumo}

A apneia do sono e o ronco primário são vinculados ao estreitamento ou obstrução das vias aéreas superiores. Entre as variadas modalidades de tratamento, estão os aparelhos intraorais que atuam controlando o colapso e a interrupção da passagem de ar. Objetivo: avaliar a evidência científica disponível sobre a eficácia dos aparelhos intraorais no tratamento do ronco primário e da Síndrome da Apneia Obstrutiva do Sono leve, moderada e grave. Revisão de Literatura: utilizou-se a estratégia de pesquisa eletrônica com palavras-chave e critério predefinido incluindo trabalhos publicados nos últimos 10 anos. Foi também utilizada a avaliação qualitativa da metodologia dos artigos. Embora um significativo número de estudos tenha analisado o assunto, apenas nove artigos foram selecionados de acordo com os critérios de inclusão e com a avaliação metodológica da elaboração do artigo. Considerações Finais: os diversos tipos de aparelhos intraorais parecem ser eficazes no controle do ronco primário e da SAOS leve e moderada, possibilitando redução dos efeitos secundários.

Palavras-chave: Apneia do sono. Ronco. Tratamento.

\section{Introdução}

O ronco é produzido pela vibração do palato mole e/ou outros tecidos orofaríngeos durante a passagem do ar, na função respiratória ${ }^{1}$. A Síndrome da Apneia e Hipopneia Obstrutiva do Sono (SAOS) é definida como o fechamento de $30 \%$, no mínimo, da via aérea nasal/bucal por 10 segundos ou mais, acompanhado de dessaturação de $4 \%$ ou mais da oxi-hemoglobina. Sua prevalência aumenta com a idade até, aproximadamente, a sétima e oitava décadas de vida, sendo mais frequente entre os homens e em mulheres na menopausa ${ }^{1-3}$.

Entre os fatores etiológicos pode-se citar: o estreitamento anatômico das vias aéreas superiores (excesso de tecidos moles, macroglossia, micro e retrognatismo) predispondo, dessa maneira, a uma elevada resistência para o fluxo de ar, a qual gera uma pressão intraluminar negativa durante a inspiração, favorecendo o colabamento da orofaringe em diferentes níveis ${ }^{1,2,4}$. Associados ao ganho de peso, aumento maior da circunferência do pescoço e ingestão de bebidas alcoólicas potencializam o colapso. Condições

Especialista em Prótese Dental - UNESP- Araraquara/SP; Mestre e Doutora em Clínica Odontológica- Área de Prótese Dental (FOP-UNICAMP) - Piracicaba/ SP; Profa. Adjunta do Curso de Odontologia - Área de Prótese e Oclusão- da Universidade Federal do Ceará- Campus Sobral - CE, Brasil.

Graduanda em Odontologia pela Universidade Federal do Ceará- Campus Sobral - CE, Brasil.

Especialista em Implantodontia (FOP-UNICAMP) - Piracicaba/ SP; Capacitação Odontológica em Medicina do Sono (Ortodontia \& Sono) - Santos/SP. Centro de Ensino em Odontologia Paulo Picanço (CEOPP) - Fortaleza-Ceará, Brasil.

* Graduada em Odontologia pela Universidade Federal do Ceará- Campus Sobral- Ceará, Brasil.

Especialista em Ortodontia; Prof. Convidado dos Cursos de Capacitação Odontológica em Medicina do Sono do CPO- SL Mandic- São Paulo/SP, Brasil. 
sistêmicas também aparecem como fatores predisponentes, tais como: a hipertensão arterial sistêmica, hipotireoidismo não tratado, acromegalia, obstrução nasal e disfunções neuromusculares ${ }^{1-4}$.

É conveniente lembrar que nem todo indivíduo que ronca possui a SAOS, assim como nem todo portador da SAOS, obrigatoriamente, ronca ${ }^{1}$.

A classificação e o diagnóstico da apneia associam-se ao índice de apneia e hipopneia (IAH), que é calculado durante o exame de Polissonografia e refere-se à quantidade de eventos apneicos pelos quais o paciente passa durante o sono. ASAOS pode ser classificadas como leves, moderadas ou severas, com IAH de 5-15, 16-30 e > 30, respectivamente ${ }^{1-3}$. É fundamental a coleta de dados por meio da história clínica, do exame físico e da realização de exames laboratoriais (polissonografia), bem como a observação da presença dos sinais e sintomas fundamentais, que são: sono não restaurador, sonolência diurna, fadiga, noctúria, alterações no desempenho cognitivo, irritabilidade, dores de cabeça matinais e movimentos anormais do corpo durante o sono $0^{1,3,5}$.

As modalidades de tratamento para a SAOS vão desde a higiene do sono, que envolve retirada do álcool e outros fármacos; adequada posição do corpo e emagrecimento, até procedimentos cirúrgicos como a glossectomia parcial, a uvulopalatofaringoplastia, e procedimentos de avanço maxilomandibular, passando pelos tratamentos clínicos com aparelho de pressão positiva contínua de ar (CPAP) e aparelhos intraorais ${ }^{4,6}$.

O padrão ouro em tratamentos envolve o procedimento de pressão aérea positiva contínua (CPAP) aplicada através de um aparelho que gera e direciona um fluxo contínuo de ar (40 a 601/min) através de um tubo flexível para uma máscara nasal firmemente aderida à face do paciente ${ }^{4}$.

Recentemente, tem-se aumentado o interesse no tratamento com aparelhos intraorais, por se tratar de uma opção simples e não invasiva ${ }^{4,6-8}$. Estes últimos podem ser de quatro tipos principais: os retentores linguais, os elevadores do palato mole, os aparelhos de pressão oral positiva e os posicionadores mandibulares. Os elevadores de palato mole têm sido pouco utilizados, pois são incômodos e não mostraram eficácia. Os retentores linguais, usados principalmente em pacientes desdentados, retêm a língua por meio de pressão negativa em um bulbo de plástico leve, mantendo-a posicionada anteriormente na cavidade oral. Aparelhos de pressão oral positiva são dispositivos que permitem a combinação do dispositivo de avanço mandibular com o sistema de pressão positiva contínua em via aérea (CPAP). Os aparelhos posicionadores mandibulares podem ter desenhos variados e mantêm a mandíbula posicionada anteriormente durante o sono, sendo estes os mais utilizados e estudados ${ }^{3,8,9}$.

Dentre os efeitos adversos dessa terapia cita-se o desconforto na articulação temporomandibular e musculatura facial, salivação ou secura excessiva da boca, aumento do ângulo do plano mandibular, diminuição de sobremordida e sobressaliência, retroinclinação dos incisivos superiores, proclinação dos incisivos inferiores, aumento da altura facial inferior, inclinação para distal dos molares superiores e inclinação mesial dos molares inferiores. Essas alterações dentoesqueléticas são progressivas e resultantes de tratamentos muito longos ${ }^{4,8,10}$.

A efetividade dos tratamentos é dada por parâmetros laboratoriais (redução do IAH, redução dos microdespertares, melhora a saturação devido ao controle da dessaturação da oxihemoglobina) e parâmetros clínicos tais como a ausência de ronco ou redução significativa desse, melhora no grau de sonolência diurna excessiva e satisfação do pacien$\mathrm{te}^{1,3,4}$.

O objetivo desta revisão de literatura consiste em avaliar a evidência científica disponível sobre a eficácia dos aparelhos intraorais no tratamento do ronco primário e da Síndrome da Apneia Obstrutiva do Sono leve, moderada e grave.

\section{Materiais e métodos}

\section{Seleção de busca}

Foi realizado uma pesquisa eletrônica nos sites PubMed (pubmed.nl), Medline (bireme.com.br) e Biblioteca Cochrane (Cochrane.bireme.br), no período compreendido entre 2005 e 2015, com os seguintes descritores nos idiomas português e inglês respectivamente: "SÍNDROME DA APNEIA DO SONO"; "RONCO"; "TRATAMENTO"; "SLEEP APNEA SYNDROMES"; "SNORING"; "TREATMENT". Inicialmente, os artigos foram selecionados pela avaliação do título associado aos critérios predefinidos, seguidos pela análise dos resumos das evidências pré-selecionadas. A busca está relatada na Figura 1.

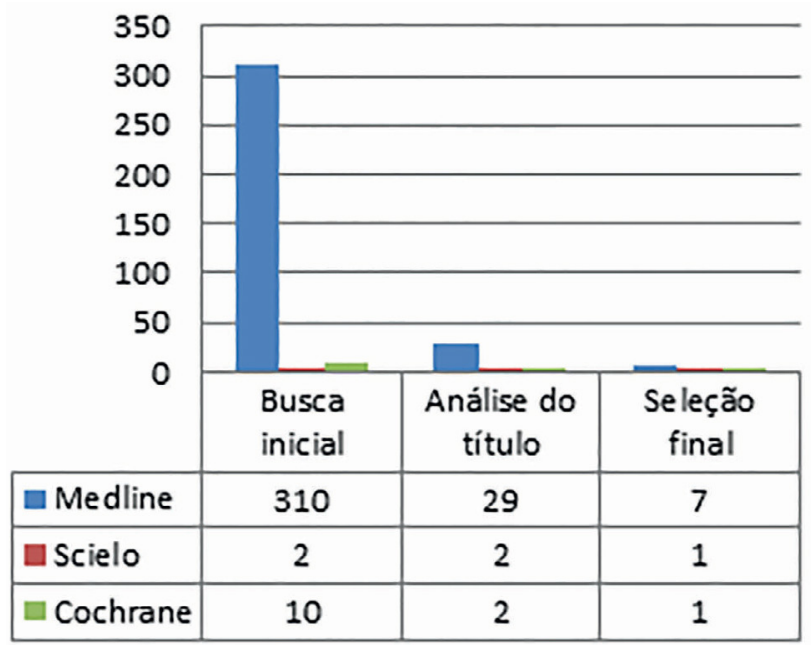

Figura 1 - Resultados encontrados durante a busca eletrônica inicial com os descritores e com o período (322 artigos), após a análise do título de acordo com os critérios de inclusão e exclusão (33 artigos) e a seleção finalizada com avaliação do resumo resultando em 09 artigos 


\section{Critérios de inclusão e exclusão escollhidos para avaliação dos artigos}

Foram utilizados como critérios de exclusão, estudos relacionados com:

1) População diagnosticada com apneia central, grave ou com outras desordens otorrinolaringológicas; Crianças e adolescentes.

2) Intervenção de outros tratamentos, exceto AIOs, como cirúrgicos, medidas preventivas e, principalmente, $o$ aparelho de pressão positiva de ar (CPAP)

3) Comparativos entre os AIOs e o CPAP, ou outras modalidades de controle.

4) Relatos de casos e estudos epidemiológicos.

Os critérios utilizados para a inclusão dos estudos consistem:

1) População diagnosticada com apneia leve e moderada e com ronco primário; Adultos de meia idade e idosos.

2) Intervenção associada ao tratamento apenas com aparelhos intraorais.

3) Comparação entre a evolução, os tipos, os efeitos colaterais e a eficácia dos diversos aparelhos intraorais.
4) Revisões de literatura, ensaios clínicos controlados ou aleatórios, em um período de 10 anos e artigos em língua portuguesa e inglesa.

\section{Seleção dos estudos}

A avaliação qualitativa da metodologia aplicada aos estudos foi realizada de acordo com os trabalhos descritos por Antczak et al. ${ }^{13}$ (1986) e Jadad et al. ${ }^{14}$ (1996). As variáveis levadas em consideração foram: tipo do estudo (estudo controlado e aleatório $=3$ pontos; estudo prospectivo $=1$ ponto; estudo retrospectivo $=0$ ); tamanho da amostra $\geq 30=1$ ponto; método correto de utilização da amostra $=1$ ponto; coerência da metodologia empregada $=1$ ponto; utilização da análise de erro do método $=1$ ponto; cegamento das medidas $=1$ ponto; testes estatísticos adequados = 1 ponto; fatores de confundimento $=1$ ponto. Cada artigo, portanto, poderia atingir a pontuação máxima de 10 pontos, sendo posteriormente classificado segundo a qualidade do trabalho (baixa $=0-5$ pontos; média $=6-8$ pontos; e alta $=$ 9-10 pontos) 4 . (Tabela 1 ).

Tabela 1 - Avaliação qualitativa dos estudos selecionados.

\begin{tabular}{|c|c|c|c|c|c|c|c|c|c|}
\hline $\begin{array}{l}\text { Autor } \\
\text { (Ano) }\end{array}$ & Tipo de Estudo & $\begin{array}{c}\text { Tamanho da } \\
\text { Amostra }\end{array}$ & $\begin{array}{l}\text { Utilização } \\
\text { da amostra }\end{array}$ & $\begin{array}{c}\text { Coerência da } \\
\text { metodologia }\end{array}$ & $\begin{array}{l}\text { Analise } \\
\text { de erro }\end{array}$ & $\begin{array}{l}\text { Cegamento } \\
\text { das medidas }\end{array}$ & $\begin{array}{c}\text { Teste } \\
\text { estatístico }\end{array}$ & $\begin{array}{c}\text { Fatores de } \\
\text { confundimento }\end{array}$ & $\begin{array}{l}\text { Qualidade } \\
\text { do trabalho }\end{array}$ \\
\hline $\begin{array}{l}\text { Levendowski et } \\
\text { al. }^{2}(2007)\end{array}$ & $\begin{array}{l}\text { Estudo clínico } \\
\text { controlado e aleatório }\end{array}$ & Adequado & Adequado & Adequado & Sim & Sim & Sim & Não & Alta \\
\hline $\begin{array}{l}\text { Deane et al. }{ }^{5} \\
\text { (2009) }\end{array}$ & $\begin{array}{l}\text { Estudo clínico } \\
\text { controlado e aleatório }\end{array}$ & Inadequado & Adequado & Adequado & Sim & Sim & Sim & Não & Alta \\
\hline $\begin{array}{l}\text { Caldas et al. }{ }^{4} \\
(2009)\end{array}$ & Estudo retrospectivo & Inadequado & Adequado & Adequando & Não & Sim & Não & Não & Baixa \\
\hline $\begin{array}{l}\text { Lee et al. }{ }^{6} \\
(2009)\end{array}$ & $\begin{array}{l}\text { Estudo clínico } \\
\text { controlado e aleatório }\end{array}$ & Adequado & Adequado & Adequado & Sim & Sim & Sim & Não & Alta \\
\hline $\begin{array}{l}\text { Martínez-Gomis } \\
\text { et al. }{ }^{7}(2010)\end{array}$ & $\begin{array}{l}\text { Estudo clínico } \\
\text { controlado e aleatório }\end{array}$ & Adequado & Adequado & Adequado & Sim & Sim & Sim & Não & Alta \\
\hline $\begin{array}{l}\text { Dieltjens et al. }{ }^{8} \\
(2013)\end{array}$ & $\begin{array}{l}\text { Estudo clínico } \\
\text { controlado e aleatório }\end{array}$ & Inadequado & Adequado & Adequado & Sim & Sim & Sim & Não & Alta \\
\hline $\begin{array}{l}\text { Lima et al. }{ }^{10} \\
(2013)\end{array}$ & $\begin{array}{l}\text { Estudo clínico } \\
\text { controlado e aleatório }\end{array}$ & Inadequado & Adequado & Adequado & Sim & Sim & Sim & Não & Alta \\
\hline $\begin{array}{l}\text { Rocha et al. }{ }^{11} \\
(2014)\end{array}$ & $\begin{array}{l}\text { Estudo clínico } \\
\text { controlado e aleatório }\end{array}$ & Inadequado & Adequado & Adequado & Sim & Sim & Sim & Não & Alta \\
\hline $\begin{array}{l}\text { Guimarães et } \\
\text { al. }^{12}(2015)\end{array}$ & Estudo retrospectivo & Inadequado & Adequado & Adequado & Não & Sim & Não & Não & Alta \\
\hline
\end{tabular}




\section{Resultados}

De acordo com os métodos qualitativos, 09 estudos foram selecionados para a revisão (Tabela 2).

Tabela 2 - Descrição dos estudos selecionados para revisão sistemática.

\begin{tabular}{|c|c|c|c|c|c|c|}
\hline $\begin{array}{l}\text { Autor } \\
\text { (ano) }\end{array}$ & Revista & Estudo & Aparelho & Atuação & Eficácia & Efeitos colaterais \\
\hline $\begin{array}{l}\text { Levendowski et } \\
\text { al. }^{2}(2007)\end{array}$ & Sleep Breath & $\begin{array}{l}\text { Ensaio clínico } \\
\text { controlado }\end{array}$ & $\begin{array}{l}\text { Dispositivo de Avanço } \\
\text { TAPII com gravador de } \\
\text { canal }\end{array}$ & $\begin{array}{l}\text { Protrusão man- } \\
\text { dibular máxima } \\
\text { com autotitu- } \\
\text { lação }\end{array}$ & $\begin{array}{l}97 \% \text { com redução de } \\
50 \% \text { do IAH ou teve } \\
\text { um } A H I \leq 10\end{array}$ & Sensibilidade \\
\hline $\begin{array}{l}\text { Deane et al. }{ }^{5} \\
(2009)\end{array}$ & SLEEP & $\begin{array}{l}\text { Ensaio clínico } \\
\text { controlado }\end{array}$ & $\begin{array}{l}\text { 1)Dispositivo de } \\
\text { avanço mandibular; 2) } \\
\text { Estabilizador de língua. }\end{array}$ & $\begin{array}{l}\text { Protrusão man- } \\
\text { dibular não titu- } \\
\text { lável; Sucção } \\
\text { lingual. }\end{array}$ & $\begin{array}{l}\text { Semelhante eficácia; } \\
91 \% \text { preferiram o de } \\
\text { avanço. }\end{array}$ & $\begin{array}{l}\text { 1)Desconforto man- } \\
\text { díbula, secura da } \\
\text { boca e irritação dos } \\
\text { tecidos moles. 2)Des- } \\
\text { conforto }\end{array}$ \\
\hline $\begin{array}{l}\text { Caldas et al. }{ }^{4} \\
(2009)\end{array}$ & $\begin{array}{l}\text { Dental Press } \\
\text { Ortodon Ortop } \\
\text { Facial }\end{array}$ & $\begin{array}{l}\text { Revisão } \\
\text { sistemática }\end{array}$ & $\begin{array}{l}\text { Aparelho de avanço } \\
\text { mandibular }\end{array}$ & $\begin{array}{l}\text { Protrusão man- } \\
\text { dibular. }\end{array}$ & $\begin{array}{l}\text { Redução: Sonolência } \\
\text { diurna; índices de ap- } \\
\text { neia e elevação de } \mathrm{O} 2 \text {. }\end{array}$ & $\begin{array}{l}\text { Desconforto muscu- } \\
\text { lar e temporomandi- } \\
\text { bular transitório }\end{array}$ \\
\hline $\begin{array}{l}\text { Lee et al. }{ }^{6} \\
(2009)\end{array}$ & $\begin{array}{l}\text { Arch Otolaryngol } \\
\text { Head Neck Surg }\end{array}$ & $\begin{array}{l}\text { Ensaio clínico } \\
\text { controlado }\end{array}$ & $\begin{array}{l}\text { Aparelho de avanço } \\
\text { mandibular }\end{array}$ & $\begin{array}{l}\text { Protrusão } \\
\text { mandibular em } \\
60 \% .\end{array}$ & $\begin{array}{l}\text { Diminuição de } 50 \% \\
\text { no IAH. }\end{array}$ & $\begin{array}{l}\text { Desconforto na ATM } \\
\text { leve. }\end{array}$ \\
\hline $\begin{array}{l}\text { Martínez-Gomis } \\
\text { et al. }{ }^{7}(2010)\end{array}$ & $\begin{array}{l}\text { Angle } \\
\text { Orthodontist }\end{array}$ & $\begin{array}{l}\text { Ensaio clínico } \\
\text { controlado }\end{array}$ & $\begin{array}{l}\text { Aparelho de avanço } \\
\text { mandibular durante } 5 \\
\text { anos. }\end{array}$ & $\begin{array}{l}\text { Protrusão } \\
\text { mandibular em } \\
70 \% .\end{array}$ & $\begin{array}{l}\text { Controla a SAOS; não } \\
\text { afeta a prevalência de } \\
\text { DTM. }\end{array}$ & $\begin{array}{l}\text { Alterações oclusais, } \\
\text { artralgia, dor miofas- } \\
\text { cial e desconforto. }\end{array}$ \\
\hline $\begin{array}{l}\text { Dieltjens et al. }{ }^{8} \\
(2013)\end{array}$ & Sleep Breath & $\begin{array}{l}\text { Ensaio clínico } \\
\text { controlado }\end{array}$ & "Custom made" & $\begin{array}{l}\text { Protrusão } \\
\text { progressiva da } \\
\text { mandíbula }\end{array}$ & $\begin{array}{l}\text { Redução na IAH para } \\
5 \text { eventos / h de sono }\end{array}$ & $\begin{array}{l}\text { Desconforto ou dor } \\
\text { dental, salivação ex- } \\
\text { cessiva e / ou sensibi- } \\
\text { lidade muscular. }\end{array}$ \\
\hline $\begin{array}{l}\text { Lima et al. }{ }^{10} \\
\text { (2013) }\end{array}$ & $\begin{array}{l}\text { Dental Press J } \\
\text { Orthod }\end{array}$ & $\begin{array}{l}\text { Ensaio clínico } \\
\text { controlado }\end{array}$ & $\begin{array}{l}\text { Tala de avanço } \\
\text { mandibular á curto } \\
\text { prazo }\end{array}$ & $\begin{array}{l}\text { Avanço mandi- } \\
\text { bular }\end{array}$ & $\begin{array}{l}60 \% \text { aceitabilidade; } \\
\text { redução do IAH. }\end{array}$ & $\begin{array}{l}\text { Não há relatos no } \\
\text { artigo }\end{array}$ \\
\hline $\begin{array}{l}\text { Rocha et al. }{ }^{11} \\
(2014)\end{array}$ & $\begin{array}{l}\text { Caderno } \\
\text { UniFAO }\end{array}$ & $\begin{array}{l}\text { Ensaio clínico } \\
\text { controlado }\end{array}$ & $\begin{array}{l}\text { Monobloco polimérico } \\
\text { com fio de } 1,0 \text { ou } \\
1,2 \mathrm{~mm}\end{array}$ & $\begin{array}{l}\text { Protrusão man- } \\
\text { dibular }\end{array}$ & $\begin{array}{l}\text { Fio } 1,2 \mathrm{~mm} \text { : posição } \\
\text { de avanço por } 6 \text { me- } \\
\text { ses inalterada }\end{array}$ & $\begin{array}{l}\text { Não há relatos no } \\
\text { artigo }\end{array}$ \\
\hline $\begin{array}{l}\text { Guimarães et } \\
\text { al. }^{12}(2015)\end{array}$ & Orthod. Sci. & $\begin{array}{l}\text { Revisão de } \\
\text { literatura }\end{array}$ & Placas lateroprotrusivas & $\begin{array}{l}\text { Avanço mandi- } \\
\text { bular e laterali- } \\
\text { dade }\end{array}$ & $\begin{array}{l}\text { Melhor adaptação, cus- } \\
\text { to e confecção. }\end{array}$ & $\begin{array}{l}\text { Não há relatos no } \\
\text { artigo }\end{array}$ \\
\hline
\end{tabular}

\section{Discussão}

O padrão-ouro para o tratamento da SAOS é a terapia da pressão positiva contínua de ar (CPAP), contudo a necessidade de colaboração dos pacientes na utilização desse mecanismo e a baixa tolerância dos mesmos ao tratamento fez com o interesse pelos AIOs tenha aumentado nos últimos anos, em virtude, de sua praticidade e dos bons resultados que têm apresentado ${ }^{4,6-8 .}$.

Como alternativa ao uso do CPAP surgiram os aparelhos intraorais para o tratamento da síndrome da apneia obstrutiva do sono (SAOS) nos primórdios da ortopedia facial dos maxilares com Pierre Robin (1934). Desde então, esses dispositivos vêm sendo reconhecidos, de maneira crescente, no tratamento e controle da SAOS e sua eficácia no tratamento desta patologia vem sendo investigada nos últimos anos ${ }^{1,5}$.
No decorrer desta revisão sistemática foram analisados 10 aparelhos quanto ao modelo, eficácia e aos efeitos colaterais, sendo que 8 aparelhos possuem o mecanismo de ação de avanço mandibular, 1 aparelho possui tanto a protrusão mandibular, quanto o movimento de lateralidade, e, apenas 1 dispositivo atua com sucção lingual. Não foram encontrados estudos nos últimos 10 anos sobre dispositivos de elevadores do palato mole. Evidências científicas sobre aparelhos de pressão oral positiva não foram selecionados para revisão em virtude do CPAP, como critério de exclusão.

Levendowski et al. ${ }^{7}$ (2007), realizaram um ensaio controlado com 30 pacientes em tratamento com aparelho intra oral, o dispositivo de Avanço TAPII com gravador de canal limitado sendo feito por encomenda, com aparelhos superior e inferior unidos por uma mecanismo de avanço na parte superior e um transversal bar ou soquete 
na parte inferior, com um macaco de parafuso controlando posição do gancho e, assim, a quantidade de protrusão, permitindo ao paciente a auto titulação utilizando uma chave até o seu limite de tolerância. A partir de um único ponto na testa e uma cânula nasal, o gravador sem fio mede a saturação de oxigênio, pulso, o fluxo de ar, esforço respiratório, os níveis de ronco, movimento da cabeça e posição da cabeça, detectou que $97 \%$ dos pacientes apresentaram pelo menos uma redução de $50 \%$ IAH ou teve um $\mathrm{AHI} \leq 10$ pós-tratamento evidenciando apenas sensibilidade durante o uso contínuo do dispositivo. Bem como, Caldas et al. ${ }^{1}$ (2009), realizaram uma revisão sistemática com 7 evidências sobre os aparelhos intraorais de avanço mandíbula concluindo que estes estão amplamente indicados para o tratamento do ronco e da SAOS como terapia principal, ou alternativa naqueles pacientes que não podem suportar a terapia da pressão positiva das vias aéreas, pois segundo os estudos, os pacientes reportaram poucos efeitos colaterais, grande tempo de uso do aparelho, desejo de continuar utilizando-o e satisfação com o tratamento, em virtude da melhora perceptível dos sintomas.

Além disso, Lee et al. ${ }^{6}$ realizaram um estudo, em 2009, avaliando o uso do dispositivo de avanço mandibular em 142 pacientes. O IAH apresentou redução para $50 \%$ em $74 \%$ dos pacientes e a taxa de sucesso em SAOS grave foi de $42 \%$ demonstrando que em casos de remissão do PAP o AIO controla não apenas a SAOS leve e a moderada, como também, a grave. Sendo relatado como efeito colateral apenas o desconforto articular, devido ao uso contínuo, contrastando com o estudo de Martínez-Gomis et al. ${ }^{8}$ (2010), que identificaram outros efeitos colaterais de grau leve nos dois primeiros anos, como artralgia, dores miofasciais, desconforto, movimentações dentárias e redução de overjet e overbyte.

Deane et al. ${ }^{5}$ (2009), por meio de um estudo com 27 pacientes objetivou avaliar a eficácia do aparelho de estabilização da língua com o aparelho de protrusão mandibular não auto titulável. $\mathrm{O}$ aparelho testado atua por meio de sucção lingual controlando o colabamento das vias aéreas superiores. $68 \%$ dos pacientes atingiram uma resposta completa ou parcial com o dispositivo de avanço mandibular, em comparação com $45 \%$ dos pacientes testados com o estabilizador da língua, como também, diminuição em IAH foi para $91 \%$ dos pacientes em comparação a $77 \%$ dos pacientes com o dispositivo teste, pois $91 \%$ preferiram o aparelho convencional, pois $86,4 \%$ relataram desconforto e remoção involuntária durante o sono com o estabilizador de língua. Os efeitos secundários causados pelo dispositivo teste foram relatados como excesso de salivação $(86,4 \%)$, secura da boca $(59,1 \%)$, e irritação dos tecidos moles (50\%) que persistiam por 3 semanas, já os efeitos colaterais gerados com o dispositivo padrão destaca-se o desconforto mandibular (59\%) e secura da boca $(50 \%)$.

Em um estudo clínico de 2013, realizado por Dieltjens et al. ${ }^{10}$, avaliou-se a eficácia de um aparelho customizado duobloc com dois cliques interligados com anexo dentais frontais que permitem titulação progressiva da mandíbula. O IAH reduziu para menos do que 5 ou $10 \mathrm{ev} / \mathrm{h}$ com uso do dispositivo. Apenas $6,6 \%$ não conseguiram continuar o tratamento por causa de desconforto ou dor dental, salivação excessiva e/ou sensibilidade muscular, o que mostra uma possibilidade de aparelho intra oral de boa adaptação por parte dos pacientes e muito efetivo.

Outrossim, novos trabalhos tem sido desenvolvidos com o objetivo de testar a eficácia e a aceitação de novas modalidades de aparelhos intra orais, como o de Lima et al. ${ }^{12}$ (2013), que propõe o uso de uma tala com um sistema de tubos de avanço, o tubipin, com faixas elásticas intermaxilares interligadas para posicionar a mandíbula, à curto prazo, ou seja, 60 dias, obtendo o resultado de que o IAH reduziu e a qualidade de sono dos pacientes com a tala melhorou em $90 \%$ dos pacientes com SAOS leve ou moderado e em $50 \%$ a $60 \%$ em pacientes com SAOS grave; o de Rocha et al. ${ }^{2}$ (2014), que por meio de um ensaio clínico controlado compararam o monobloco polimérico com fio 1.2 e com fio 1.0 tradicional concluindo que a resistência do fio aumentou, consideravelmente, permitindo por 6 meses a manutenção da posição de titulação do dispositivo intra oral; e, finalmente, o de Guimarães et al. ${ }^{3}$ (2015), que através de uma revisão sistemática apresentaram as placas lateroprotrusivas para tratamento da SAOS. Sistema que é constituído por duas placas acrílicas aderidos por grampos de retenção interligadas por parafusos e expansores que possibilitam avanços graduais da mandíbula com movimentos de lateralidade, no intuito, de reduzir desconfortos articulares e musculares.

É evidente que além da redução dos IAH, todos os dispositivos testados controlaram a saturação de oxigênio, reduziram o ronco primário e todos os sinais e sintomas referentes a SAOS, como também, em casos específicos controlam a SAOS grave ${ }^{6,12}$. Os efeitos secundários evidenciados são contornáveis e não prejudicam a manutenção da terapia e a qualidade de vida do paciente ${ }^{6,8,10}$.

Além dos estudos citados, Caram et al. ${ }^{15}$ (2013) em 1993 idealizaram uma nova modalidade terapêutica, apresentada à comunidade científica em 2013, com o objetivo de superar as limitações dos retentores linguais existentes no que tange ao controle da SAOS grave, e que vem mostrando excelentes resultados, conhecida como Técnica Lingual de Caram. A técnica é composta por dois aparelhos. O primeiro deles, designado como Órtese Lingual ou simplesmente "Mola" consiste em uma única pla- 
ca, de encaixe maxilar, através da qual deriva-se o acessório para o controle lingual. $\mathrm{O}$ segundo aparelho consiste em um aparelho bibloco, articulado por meio de arcos vestibulares helicoidais, que funciona por meio do avanço mandibular associado à ação mecânica direta sobre a língua. Em alguns casos, a órtese poderá excluir o tradicional reposicionamento mandibular e atuar somente no controle ativo da posição lingual. Cabe ressaltar que a ação ideal do mecanismo somente é alcançada após sessões de titulação ${ }^{15}$. Este aparelho tem sido bastante estudado e vem apresentando resultados promissores até mesmo no controle da SAOS grave, por promover um efetivo aumento do espaço aéreo.

\section{Conclusão}

Através da análise dos trabalhos selecionados para a revisão sistemática é possível concluir que os aparelhos intraorais parecem ser eficazes no controle do ronco primário, da SAOS leve e moderada, e até mesmo da SAOS grave, como relatado por alguns autores, possibilitando redução dos sinais e sintomas associados ao uso contínuo do dispositivo. No entanto a quantidade de artigos a respeito do assunto é pequena e são necessários mais estudos utilizando comparativos, como placebo ou outras terapias, que possuam um tempo de proservação maior para avaliar a importância dos efeitos adversos desse tipo de terapia, como distúrbios articulares e má-oclusão.

\section{Abstract}

Sleep apnea and primary snoring are related to the narrowing or obstruction of the upper airways. Among several treatment modes are intraoral appliances, which control the collapse and obstruction of the air passageway. Objective: to assess the available scientific evidence on the effectiveness of intraoral appliances in treating primary snoring, as well as mild, moderate, and severe obstructive sleep apnea syndrome. Literature Review: an electronic search strategy was used with keywords and predefined criteria including papers published over the last 10 years. The qualitative assessment of study methods was also used. Although a significant number of studies have examined the issue, only nine articles were selected according to the inclusion criteria and the methodological assessment of article preparation. Final Considerations: the several types of intraoral appliances seem effective for controlling primary snoring and mild to moderate OSAS, allowing the reduction of side effects.

Keywords: Sleep apnea. Snoring. Treatment.

\section{Referências}

1. Caldas SGFR, Ribeiro AA, Pinto LS, Martins LP, Matoso RM. Efetividade dos aparelhos intrabucais de avanço mandibular no tratamento do ronco e da síndrome da apneia e hipopneia obstrutiva do sono (SAOS): revisão sistemática. R Dental Press Ortodon Ortop Facial 2009;14(4):74-82.

2. Rocha GM, Bicalho LA, Magnago RO. Seleção de fio de aço inox utilizado na haste do aparelho intraoral de pacientes em tratamento de ronco e apneia do sono. Cadernos UniFao - Edição Especial do Curso de Mestrado Profissional em Materiais - 2014 dezembro.

3. Guimarães MLR, Oliveira JJM, Azevedo PG. Aparelho PLP para tratamento de ronco e apneia obstrutiva do sono. Orthod Sci Pract 2015; 8(29):113-7.

4. Almeida MAO, Teixeira AOB, Vieira LS, Quintão CCA. Tratamento da síndrome da apnéia e hipopnéia obstrutiva do sono com aparelhos intrabucais. Rev Bras Otorrinolaringol 2006; 72(5):699-703.

5. Deane SA, Cistulli PA, Zeng B, Petocz P, Darendeliler MA Comparison of mandibular advancement splint and tongue stabilizing device in obstructive sleep apnea: a randomized controlled trial. SLEEP 2009; 32(5):648-53.

6. Lee CH, Mo JH, Choi IJ, Lee JH, Seo BS, Kim DY, et al. The Mandibular Advancement Device and Patient Selection in the Treatment of Obstructive Sleep Apnea. Arch Otolaryngol Head Neck Surg. 2009; 135(5):439-44.

7. Levendowski DJ, Morgan TD, Patrickus JE, Westbrook PR, Berka C, Zavora T, et al. In-home evaluation of efficacy and titration of a mandibular advancement device for obstructive sleep apnea. Sleep Breath 2007; 11:13947 .

8. Martínez-Gomisa J, Willaerta E, Noguesa L, Pascuala M, Somozab M, Monasterioc C. Five Years of Sleep Apnea Treatment with a Mandibular Advancement Device Side Effects and Technical Complications. Angle Orthod 2010; 80:30-6.

9. Doff MHJ, Finnema KJ, Hoekema A, Wijkstra PJ, Bont LGM, Stegenga B. Long-term oral appliance therapy in obstructive sleep apnea syndrome: a controlled study on dental side effects. Clin Oral Invest 2013; 17:475-82.

10. Dieltjens M, Vanderveken OM, Hamans E, Verbraecken JA, Wouters K, Willemen M, et al. Treatment of obstructive sleep apnea using a custom-made titratable duobloc oral appliance: a prospective clinical study. Sleep Breath 2013; $17: 565-72$

11. Schwarting S, Huebers U, Heise M, Schlieper J, Hauschil A. Position paper on the use of mandibular advancement devices in adults with sleep-related breathing disorders A position paper of the German Society of Dental Sleep Medicine (Deutsche Gesellschaft Zahnaerztliche Schlafmedizin, DGZS). Sleep Breath 2007; 11:125-6.

12. Lima CMP, Furquim LZ, Ramos AL. Short-term efficacy of mandibular advancement splint in treatment of obstructive sleep apnea hypopnea syndrome. Dental Press J Orthod $2013 ;$ 18(3):118-23.

13. Antczak AA, Tang J, Chalmers TC. Quality assessment of randomized control trials in dental research. J Periodontal Res 1986 ; 21(4):305-14.

14. Jadad AR, Moore RA, Carroll D, Jenkinson C, Reynolds DJ, Gavaghan DJ, et al. Assessing the quality of reports of randomized clinical trials: is blinding necessary? Control Clin Trials, $1996 ; 17(1): 1-12$. 
15. Caram JM, Quintela MM, et al. A órtese lingual - uma nova proposta de aparelho intraoral para apneia do sono grave relato de casos. Orthod Sci Pract. 2013; 6:110-7.

\section{Endereço para correspondência:}

Millena Teles Portela de Oliveira.

Rodovia Vilebaldo Aguiar № 978.

62.160.000 COREAU-CEARA, Brasil

Fone: (88)36451666/(88)988016882

E-mail: millenateen2@hotmail.com

Recebido: 09/08/2016. Aceito: 04/08/2017. 